ADP-03-128/T564

DESY 03-157

\title{
Electromagnetic Form Factors with FLIC fermions
}

\author{
J. M. Zanotti ${ }^{\text {ab } *}$, D. B. Leinweber ${ }^{\mathrm{a}}$, A. G. Williams ${ }^{\mathrm{a}}$ and J. B. Zhang ${ }^{\mathrm{a}}$

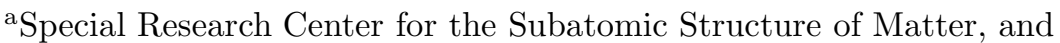 \\ Department of Physics, University of Adelaide Adelaide SA 5005 Australia \\ ${ }^{b}$ John von Neumann-Institut für Computing NIC, \\ Deutsches Elektronen-Synchrotron DESY, D-15738 Zeuthen, Germany
}

The Fat-Link Irrelevant Clover (FLIC) fermion action provides a new form of nonperturbative $\mathcal{O}(a)$ improvement and allows efficient access to the light quark-mass regime. FLIC fermions enable the construction of the nonperturbatively $\mathcal{O}(a)$-improved conserved vector current without the difficulties associated with the fine tuning of the improvement coefficients. The simulations are performed with an $\mathcal{O}\left(a^{2}\right)$ mean-field improved plaquetteplus-rectangle gluon action on a $20^{3} \times 40$ lattice with a lattice spacing of $0.128 \mathrm{fm}$, enabling the first simulation of baryon form factors at light quark masses on a large volume lattice. Magnetic moments, electric charge radii and magnetic radii are extracted from these form factors, and show interesting chiral nonanalytic behavior in the light quark mass regime.

\section{INTRODUCTION}

The magnetic moments of baryons have been identified [12] as providing an excellent opportunity for the direct observation of chiral nonanalytic behavior in lattice QCD, even in the quenched approximation. This paper will present results for baryon electromagnetic structure in which the chiral nonanalytic behaviour predicted by quenched chiral perturbation theory is observed in the numerical simulation results.

FLIC fermions provide a new form of nonperturbative $\mathcal{O}(a)$ improvement 34 where nearcontinuum results are obtained at finite lattice spacing. Access to the light quark mass regime is enabled by the improved chiral properties of the lattice fermion action 4. A key feature of the FLIC fermion approach is that the fine tuning of $\mathcal{O}(a)$-improvement term coefficients is not necessary. The improvement terms are irrelevant operators which are constructed using fat-links. Remaining perturbative renormalizations are accurately accounted for by small mean-field improvement corrections. Hence, we are able to determine the form factors of octet and decuplet baryons with unprecedented accuracy.

\footnotetext{
*Presented by J. M. Zanotti at Lattice '03
}

\section{LATTICE ACTIONS}

The simulations are performed using a meanfield $\mathcal{O}\left(a^{2}\right)$-improved Luscher-Weisz [5] gauge action on a $20^{3} \times 40$ lattice with a lattice spacing of $0.128 \mathrm{fm}$ as determined by the Sommer scale $r_{0}=0.50 \mathrm{fm}$. We use a minimum of 255 configurations and the error analysis is performed by a third-order, single-elimination jackknife.

Fat links are created using APE smearing [6] followed by projection of the smeared link back to $S U(3)$. We select a smearing fraction of $\alpha=$ 0.7 (keeping 0.3 of the original link) and iterate the process six times [7]. Further details of FLIC fermion actions can be found in Ref. 8].

For fat links, the mean link $u_{0} \approx 1$, enabling the use of highly improved definitions of the lattice field strength tensor, $F_{\mu \nu}$ 9. In particular, we employ an $\mathcal{O}\left(a^{4}\right)$-improved definition of $F_{\mu \nu}$ in which the standard clover-sum of four $1 \times 1$ Wilson loops lying in the $\mu, \nu$ plane is combined with $2 \times 2$ and $3 \times 3$ Wilson loop clovers. Moreover, mean-field improvement of the coefficients of the clover and Wilson terms of the fermion action is sufficient to accurately match these terms and eliminate $\mathcal{O}(a)$ errors from the fermion action. As the conserved vector current has its origin in the fermion action, mean-field improvement of the ir- 
relevant operator terms is also sufficient to accurately remove $\mathcal{O}(a)$ errors, providing a nonperturbatively $\mathcal{O}(a)$-improved conserved vector current.

For the construction of the $\mathcal{O}(a)$-improved conserved vector current, we follow the technique proposed by Martinelli et al. 12. The standard conserved vector current for Wilson-type fermions is derived via the Noether procedure

$$
\begin{aligned}
j_{\mu}^{\mathrm{C}} & \equiv \frac{1}{4}\left[\bar{\psi}(x)\left(\gamma_{\mu}-r\right) U_{\mu}(x) \psi(x+\hat{\mu})\right. \\
& +\bar{\psi}(x+\hat{\mu})\left(\gamma_{\mu}+r\right) U_{\mu}^{\dagger}(x) \psi(x) \\
& +(x \rightarrow x-\hat{\mu})] .
\end{aligned}
$$

The $\mathcal{O}(a)$ improvement term is also derived from the fermion action and is constructed in the form of a total four-divergence, preserving charge conservation. The $\mathcal{O}(a)$-improved conserved vector current is

$j_{\mu}^{\mathrm{CI}} \equiv j_{\mu}^{\mathrm{C}}(x)+\frac{r}{2} C_{C V C} a \sum_{\rho} \partial_{\rho}\left(\bar{\psi}(x) \sigma_{\rho \mu} \psi(x)\right),(2)$

where $C_{C V C}$ is the improvement coefficient for the conserved vector current and we define

$\partial_{\rho}(\bar{\psi}(x) \psi(x)) \equiv \bar{\psi}(x)\left(\overleftarrow{\nabla}_{\rho}+\vec{\nabla}_{\rho}\right) \psi(x)$.

The terms proportional to the Wilson parameter $r$ in Eq. (11) and the four-divergence in Eq. (2) have their origin in the irrelevant operators of the fermion action and vanish in the continuum limit. Nonperturbative improvement is achieved by constructing these terms with fat-links. As we have stated, perturbative corrections are small for fat-links and the use of the tree-level value for $C_{C V C}=1$ together with small mean-field improvement corrections ensures that $\mathcal{O}(a)$ artifacts are accurately removed from the vector current. This is only possible when the current is constructed with fat-links. Otherwise, $C_{C V C}$ needs to be appropriately tuned to ensure all $\mathcal{O}(a)$ artifacts are removed.

A fixed boundary condition at $t=0$ is used for the fermions and gauge-invariant Gaussian smearing [1011] in the spatial dimensions is applied at the source at $t=8$ to increase the overlap of the interpolating operators with the ground state while suppressing excited state contributions. The technique used for constructing the

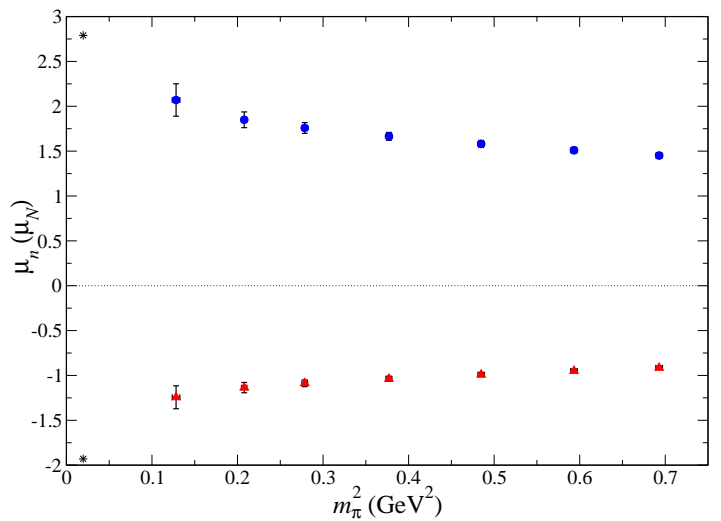

Figure 1. FLIC fermion simulation results for the magnetic moments of the proton ( $\circ$ ) and neutron $(\triangle)$ in quenched QCD.

three-point functions follows the procedure outlined in detail in Refs. 1314. In particular, we use the sequential source technique at the current insertion. Correlation functions are made purely real with exact parity through the consideration of $U$ and $U^{*}$ link configurations. Electric and magnetic form factors are extracted by constructing ratios of two- and three-point functions. We simulate at the smallest finite $q^{2}$ available on our lattice, $\vec{q}=\frac{2 \pi}{a L} \widehat{x}$.

\section{RESULTS}

Figure 1 displays FLIC fermion simulation results for the magnetic moments of the proton and neutron in quenched QCD. At heavy quark masses we note that the magnetic moments of both nucleons display linear behaviour when plotted as a function of $m_{\pi}^{2}$. As one approaches the light quark mass regime, we find evidence of nonanalytic behaviour in the nucleon magnetic moments as predicted by quenched $\chi \mathrm{PT}[12]$. In fact, if we were to flip the sign of the neutron magnetic moment, we would find that the proton and neutron have a very similar behaviour as a function of $m_{\pi}^{2}$ in the light quark mass regime as predicted by the leading non-analytic contributions of quenched $\chi \mathrm{PT}$.

Figure 2 displays results for the proton charge radius obtained from a dipole form factor ansatz. Some curvature is emerging as the chiral limit is 


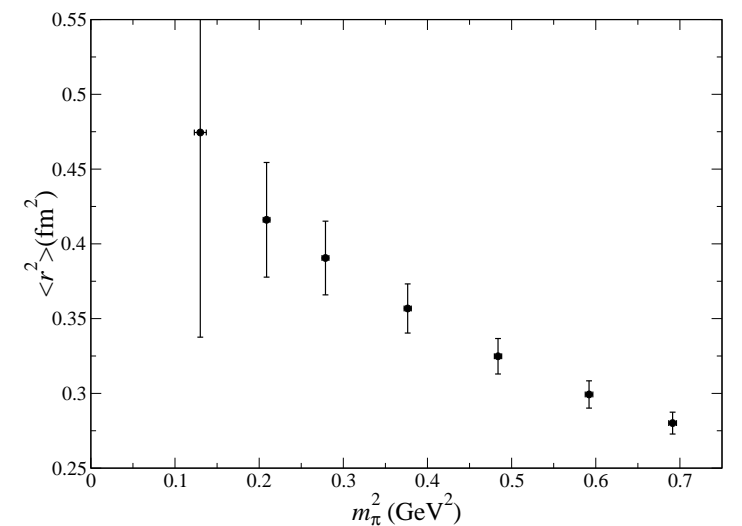

Figure 2. FLIC fermion simulation results for the charge radius of the proton in quenched QCD.

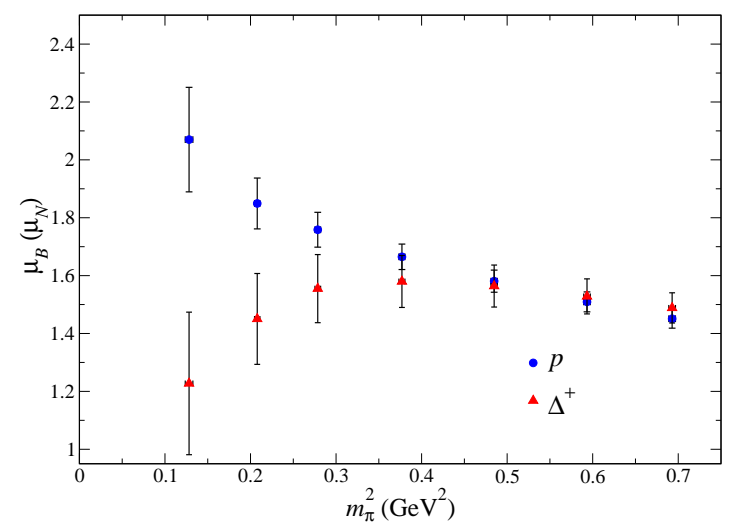

Figure 3. FLIC fermion simulation results for the magnetic moments of the proton (o) and $\Delta^{+}$resonance $(\triangle)$ in quenched QCD.

approached.

Figure 3 displays FLIC fermion simulation results for the magnetic moments of the proton and $\Delta^{+}$resonance in quenched QCD. At large pion masses, the $\Delta$ moment is enhanced relative to the proton moment in accord with earlier lattice QCD results 1314 and model expectations. However as the chiral regime is approached the nonanalytic behavior of the quenched meson cloud is revealed, enhancing the proton and suppressing the $\Delta^{+}$in accord with the expectations of quenched $\chi \mathrm{PT}$ [15 16. The quenched artifacts of the $\Delta$ provide an unmistakable signal for the onset of quenched chiral nonanalytic behavior.

We have presented the first lattice QCD simula- tion results for the electromagnetic form factors of the nucleon and $\Delta$ at quark masses light enough to reveal unmistakable quenched chiral nonanalytic behavior.

This work was supported by the Australian Research Council. We thank the Australian Partnership for Advanced Computing (APAC) for generous grants of supercomputer time which have enabled this project.

\section{REFERENCES}

1. D. B. Leinweber, Nucl. Phys. Proc. Suppl. 109 (2002) 45 hep-lat/0112021.

2. D. B. Leinweber, hep-lat/0211017

3. D. B. Leinweber, et al., nucl-th/0211014

4. J. Zanotti, et al., in preparation.

5. M. Luscher and P. Weisz, Commun. Math. Phys. 97, 59 (1985) [ibid. 98, 433 (1985)].

6. M. Falcioni et al., Nucl. Phys. B251 (1985) 624; M. Albanese et al., Phys. Lett. B 192 (1987) 163.

7. F.D. Bonnet et al., Phys. Rev. D 62 (2000) 094509 hep-lat/0001018.

8. J.M. Zanotti et al., Phys. Rev. D 60 (2002) 074507 hep-lat/0110216; Nucl.Phys.Proc.Suppl. $\quad \mathbf{1 0 9} 101 \quad$ (2002) hep-lat/0201004.

9. S. O. Bilson-Thompson, D. B. Leinweber and A. G. Williams, Annals Phys. 304 (2003) 1 hep-lat/0203008.

10. S. Gusken, Nucl. Phys. Proc. Suppl. 17, 361 (1990).

11. J. M. Zanotti, et al., to appear in Phys. Rev. D, hep-lat/0304001

12. G. Martinelli, C. T. Sachrajda and A. Vladikas, Nucl. Phys. B 358 (1991) 212.

13. D. B. Leinweber, R. M. Woloshyn and T. Draper, Phys. Rev. D 43 (1991) 1659.

14. D. B. Leinweber et al., Phys. Rev. D 46 (1992) 3067 hep-lat/9208025.

15. D. B. Leinweber, et al., nucl-th/0308083

16. R. D. Young, et al., hep-lat/0309187. 